\title{
Dysregulation of the MiR-449b target TGFBI alters the TGF $\beta$ pathway to induce cisplatin resistance in nasopharyngeal carcinoma
}

\author{
Pierre-Antoine Bissey ${ }^{1}$, Jacqueline H. Law ${ }^{2}$, Jeff P. Bruce', Wei Shi' ${ }^{1}$ Aline Renoult ${ }^{3}$, Melvin L. K. Chua $\mathbb{B}^{1,4,5,6}$,
} Kenneth W. Yip ${ }^{1}$ and Fei-Fei Liu ${ }^{1,2,4}$

\begin{abstract}
Despite the improvement in locoregional control of nasopharyngeal carcinoma (NPC), distant metastasis (DM), and chemoresistance persist as major causes of mortality. This study identified a novel role for miR-449b, an overexpressed gene in a validated four-miRNA signature for NPC DM, leading to chemoresistance via the direct targeting of transforming growth factor beta-induced (TGFBI). In vitro shRNA-mediated downregulation of TGFBI induced phosphorylation of PTEN and AKT, increasing cisplatin resistance. Conversely, the overexpression of TGFBI sensitized the NPC cells to cisplatin. In NPC patients treated with concurrent chemoradiotherapy (CRT), the overall survival (OS) was significantly inversely correlated with miR-449b, and directly correlated with both TGFBI mRNA and protein expression, as assessed by RNA sequencing and immunohistochemistry (IHC). Mechanistically, coimmunoprecipitation demonstrated that TGFBI competes with pro-TGF $\beta 1$ for integrin receptor binding. Decreased TGFBI led to increased pro-TGF $\beta 1$ activation and TGF $\beta 1$ canonical/noncanonical pathway-induced cisplatin resistance. Thus, overexpression of miR-449b decreases TGFBI, thereby altering the balance between TGFBI and pro-TGF $\beta 1$, revealing a novel mechanism of chemoresistance in NPC.
\end{abstract}

\section{Introduction}

Nasopharyngeal carcinoma (NPC) is an Epstein-Barr virus (EBV)-associated malignancy ${ }^{1,2}$ that accounts for 87,000 new cases and 51,000 deaths annually ${ }^{3,4}$. Patients with locally advanced disease have a 5 -year overall survival (OS) rate of only about $65 \%{ }^{5}$. While treatment with intensity-modulated radiation therapy (RT) has significantly improved locoregional control, distant metastasis (DM) remains a major clinical challenge, causing death in $20-30 \%$ of all NPC patients ${ }^{6}$. Furthermore, the therapeutic options for patients with locally advanced NPC remain limited, and resistance to chemoradiation

Correspondence: F-F. Liu (Fei-Fei.Liu@rmp.uhn.on.ca)

${ }^{1}$ Princess Margaret Cancer Centre, University Health Network, Toronto, ON, Canada

${ }^{2}$ Department of Medical Biophysics, University of Toronto, Toronto, ON, Canada

Full list of author information is available at the end of the article remains a major clinical challenge ${ }^{7}$. Concurrent cisplatin or 5-fluorouracil chemotherapy in combination with RT provides only a modest improvement in OS, yet causes significant toxicity and sometimes even death ${ }^{5,6,8-10}$. Hence, there is an urgent need to better understand the underlying factors that drive DM and treatment resistance, which to date have remained elusive.

The transforming growth factor beta (TGF $\beta$ ) pathway has been recently implicated in chemoresistance. Mechanistically, this process may occur via either an epithelial-to-mesenchymal transition $(\mathrm{EMT})^{11-13}$ or the maintenance of tumor-initiating cell heterogeneity ${ }^{14}$. Transforming growth factor beta 1 (TGF $\beta 1$ ) is secreted as part of a large latent complex (LLC), which binds to, and is stored in the extracellular matrix (ECM). The LLC is composed of the mature TGF $\beta 1$ non-covalently bound to its latency-associated propeptide, forming small latent complex, which in turn is covalently attached to the large

\section{(c) The Author(s) 2018}

(c) (i) Open Access This article is licensed under a Creative Commons Attribution 4.0 International License, which permits use, sharing, adaptation, distribution and reproduction cc) in any medium or format, as long as you give appropriate credit to the original author(s) and the source, provide a link to the Creative Commons license, and indicate if changes were made. The images or other third party material in this article are included in the article's Creative Commons license, unless indicated otherwise in a credit line to the material. If material is not included in the article's Creative Commons license and your intended use is not permitted by statutory regulation or exceeds the permitted use, you will need to obtain permission directly from the copyright holder. To view a copy of this license, visit http://creativecommons.org/licenses/by/4.0/. 


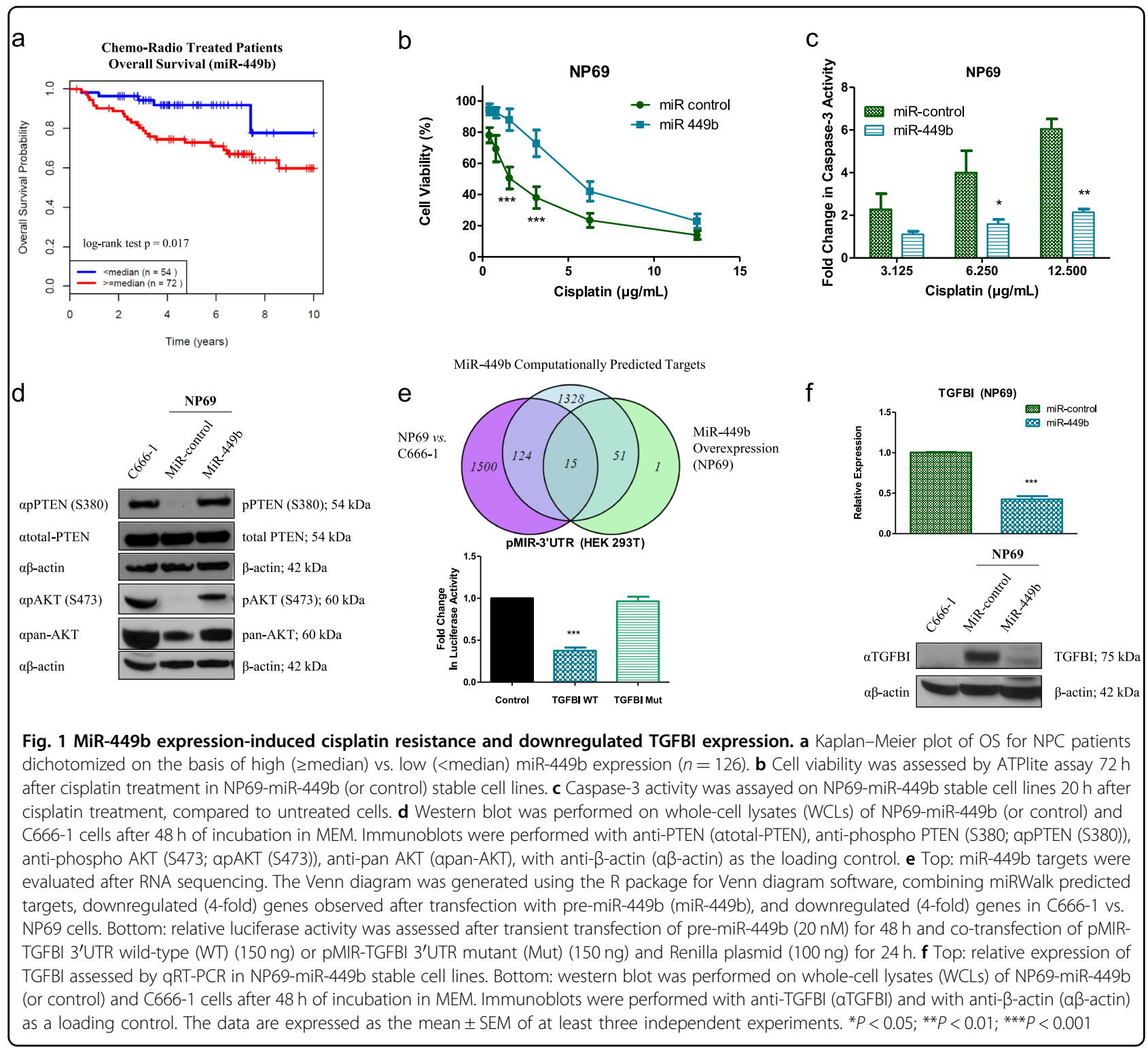

latent TGF $\beta 1$-binding $\operatorname{protein}^{15}$. In the ECM, the LLC may be subsequently activated when its Arg-Gly-Asp (RGD) motif binds directly to the RGD motif of the integrin receptors (e.g., $\alpha v \beta 3, \alpha v \beta 5$, and $\alpha v \beta 6$ ), and the mechanical forces exerted between the ECM and integrins trigger the release of active TGF $\beta 1^{16,17}$.

Among a plethora of TGF $\beta$ targets (reviewed in ref. ${ }^{18}$ ) is transforming into growth factor beta-induced (TGFBI), a secreted protein containing four fasciclin 1 domains and an RGD motif that facilitates its interaction with integrins (i.e., $\alpha v \beta 3$ and $\alpha v \beta 5)^{19-24}$. Similar to TGF $\beta 1$, both oncogenic and tumor suppressor functions have been reported for TGFBI ${ }^{25}$. Disruption of TGFBI function can lead to spontaneous tumor formation ${ }^{26}$ and resistance to chemotherapy ${ }^{27-30}$. Furthermore, TGFBI overexpression can trigger apoptosis and inhibit angiogenesis ${ }^{19,31-33}$. In several malignancies, however, TGFBI expression was observed to be elevated compared to normal tissues ${ }^{34,35}$, hence, the role of TGFBI appears to be highly dependent on the cellular context. While the TGF $\beta$ pathway has been extensively studied, the mechanisms responsible for mediating apoptosis vs. survival have not yet been completely elucidated.

Our laboratory recently completed a global microRNA (miRNA) profiling of two independent NPC cohorts, identifying and validating a four-miRNA (miR-34c, miR140, miR-154, and miR-449b) prognostic signature for $\mathrm{DM}^{36}$. However, the mechanisms by which these miRNAs affect NPC biology remained to be determined. In the present study, we examined the functional role of miR- 
449b in NPC and identified a new miR-449b TGFBI axis as a key regulator of the TGF $\beta$ pathway. Our findings demonstrate that miR-449b directly binds and represses TGFBI mRNA, altering the TGFBI-TGF $\beta 1$ balance to facilitate activation of the TGF $\beta$ pathway, thereby inducing treatment resistance in NPC.

\section{Results}

\section{MiR-449b induces cisplatin resistance and targets TGFBI}

Given the significance of metastasis and chemoresistance in $\mathrm{NPC}^{37-40}$, the four-miRNA DM prognostic signature was examined in further detail for its associations with chemoresistance ${ }^{36}$. As shown in Fig. 1a, chemoradiotherapy (CRT)-treated NPC patients with elevated miR-449b expression experienced an inferior 5 -year OS of 72.8 vs. $91.8 \%$ for low miR-449b-expressing patients ( $p=$ 0.017; the median follow-up time for these patients was 6.09 years, with a minimum of 0.96 years and a maximum of 13.5 years). This observation motivated a further examination of the role of miR-449b in vitro. C666-1, an EBV-positive NPC cell line, exhibited higher endogenous miR-449b, compared to both NP69 and NP460, two immortalized normal nasopharyngeal cell lines (Supplementary Figure S1a). Since the most common chemotherapeutic agent used in NPC is cisplatin ${ }^{7,41}$, it was hypothesized that miR-449b might be responsible for drug resistance. Indeed, C666-1 cells were more resistant to cisplatin, compared to both NP69 and NP460, as assessed by cell viability (Supplementary Figure S1b) and caspase-3 activity (Supplementary Figure S1c). NP69 cells stably transfected with miR-449b (NP69-miR-449b) recapitulated the increased cell viability (Fig. 1b) and lower caspase- 3 activity (Fig. 1c) in response to cisplatin, compared to the control cells stably transfected with scrambled miRNA (NP69-miR-control).

In order to identify the signaling pathway that might contribute to cisplatin resistance observed in NP69-miR449b and C666-1 cell lines, key proteins of wellestablished molecular pathways involved in NPC were investigated including the ERK1/2, SAPK/JNK, and PTEN/AKT pathways ${ }^{42}$. As can be seen in Supplementary Figure S1d and e, C666-1 cells exhibited higher ERK1/2 and SAPK/JNK activity, compared to normal NP69 and NP460 cells, but no significant changes were observed in NP69-miR-449b cells. The expression of p38 was also assessed, but phosphorylation was not detected by western blot. Greater levels of PTEN tail phosphorylation (Ser380) (Fig. 1d, Supplementary Figure S1f, Supplementary Figure S5a), which has been reported to inactivate PTEN by inducing a closed conformation ${ }^{43,44}$, were observed in both C666-1 and NP69-miR-449b cells. This closed conformation was indeed accompanied by higher levels of phospho-AKT (Ser473) (Fig. 1d, and Supplementary Figure S1f, Supplementary Figure S5a), suggesting one mechanism by which chemoresistance is induced in NPC cells. Indeed, the treatment of cisplatinresistant NP69-miR-449b cells with MK-2206, an AKT inhibitor, restored the sensitivity to cisplatin (to a level similar of their control; Supplementary Figure S1g).

In order to identify candidate mRNA targets of miR449b, NP69 cells were transfected with pre-miR-449b, followed by RNA sequencing using the Illumina HiSeq 2000. At the intersection of: (1) genes downregulated in the transfected cells; (2) computationally predicted targets; and (3) genes downregulated in C666-1 (high endogenous miR-449b) vs. NP69 (low endogenous miR449b) cells were 15 genes (Fig. 1e, top, Supplementary Figure S2a). Amongst these genes was TGFBI, which when upregulated, has been reported to decrease AKT activity and induce chemosensitivity ${ }^{27,28,30,45}$. Based on these observations, we decided to focus our study on TGFBI and its potential role in NPC chemoresistance. First, direct targeting of TGFBI by miR-449b was confirmed using a luciferase reporter assay (Fig. 1e, bottom). These findings were further corroborated as NP69-miR$449 \mathrm{~b}$ cells expressed lower levels of TGFBI, as observed by both quantitative real-time PCR (qRT-PCR) (Fig. 1f, top; Supplementary Figure S2b; transient transfection), as well as western blotting for cytosolic (Fig. 1f, bottom, Supplementary Figure S5a) and secreted (Supplementary Figure S2c) protein expression, when compared to NP69miR-control. Inhibition of miR-449b led to an increase of TGFBI expression at both the RNA level (Supplementary Figure S2d, top, stable cells; bottom, transient transfection), and protein level: cytosolic (Supplementary Figure S2e, left) and secreted (Supplementary Figure S2e, right). Moreover, NP69 cells stably expressing a miR-449b inhibitor (NP69-anti-miR-449b) had increased the sensitivity to cisplatin (Supplementary Figure S2f), confirming that miR-449b, and potentially TGFBI, has a role in cisplatin resistance in NPC cells.

\section{TGFBI increases cisplatin sensitivity in NP69 cells}

In order to further evaluate the role of TGFBI in cisplatin sensitivity, NP69 cells were stably infected with lentiviral vectors containing two different shRNAs targeting TGFBI (NP69-shTGFBI-1 and NP69-shTGFBI-2) (Fig. 2a, Supplementary Figures S2g and h, Supplementary Figure S5b). Both cell lines exhibited increased clonogenicity and cell viability after cisplatin treatment, when compared to the controls (Fig. 2b, c). Furthermore, both cell lines exhibited decreased caspase-3 activity following cisplatin treatment (Fig. 2d). TGFBI repression induced phosphorylation (Ser380) and inhibition of PTEN, as well as phosphorylation (Ser473) and activation of AKT (Fig. 2e, Supplementary Figure S5b). These data corroborate the results observed with miR-449b overexpression (Fig. 


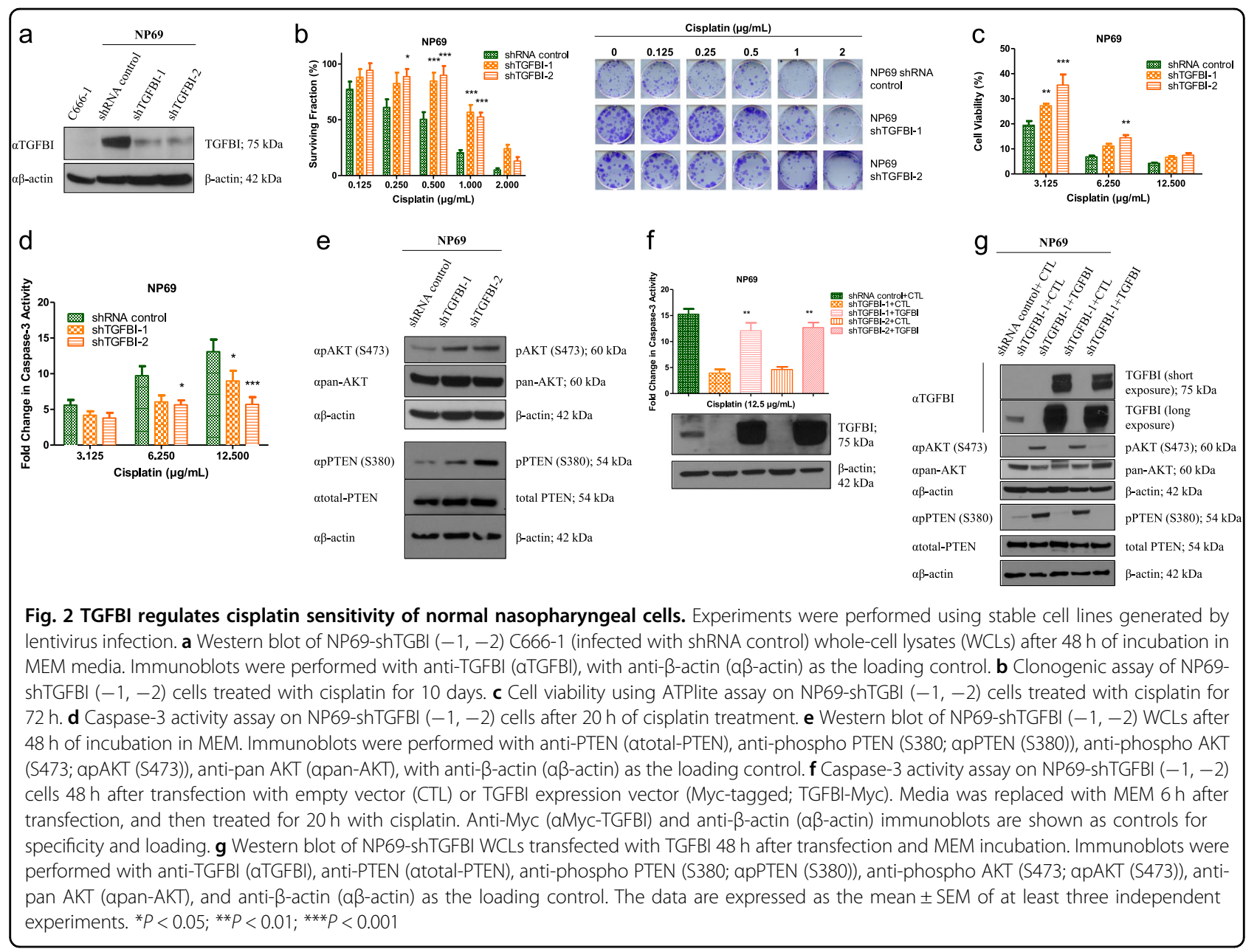

1), suggesting a pivotal role of TGFBI inhibition in miR449b-mediated cisplatin resistance.

Re-expression of TGFBI in NP69-shTGFBI cells restored their sensitivity to cisplatin to a similar level as NP69 control cells (Fig. 2f). In addition, TGFBI reexpression in NP69-shTGFBI cells abolished PTEN phosphorylation, leading to PTEN activation and reduced downstream AKT phosphorylation (Fig. 2g, Supplementary Figure S5c). Taken together, these data indicate that TGFBI modulates their sensitivity to cisplatin via regulation of the PTEN-AKT pathway.

\section{TGFBI triggers apoptosis and restores cisplatin sensitivity in NPC}

Since TGFBI is not endogenously expressed in C666-1 cells (Fig. 3a, and Supplementary Figures S3a and b), this model was utilized to assess the consequences of TGFBI overexpression. As anticipated, TGFBI overexpression in C666-1 cells triggered caspase-3 activation (Fig. 3b), corroborating the previously published results demonstrating the pro-apoptotic properties of TGFBI $^{32,33}$. Concordantly, TGFBI overexpression sensitized C666-1 cells to cisplatin (Fig. 3c, d; C666-1 cells are not suitable for clonogenic assays as they do not form colonies), activated PTEN (through reduced phospho-PTEN Ser380), and reduced AKT phosphorylation (low phospho-AKT-S473; Fig. 3e, Supplementary Figure S5d).

In order to investigate the clinical significance of TGFBI in NPC, RNA sequencing was performed on the diagnostic biopsy samples from NPC patients treated with CRT $(n=20)$. As anticipated, patients with elevated TGFBI experienced a superior 5-year distant relapse-free survival (DRFS) of 90 vs. $40 \%$ for patients with low TGFBI expression ( $p=0.082$; Fig. $3 \mathrm{f})$. This observation was further confirmed using immunohistochemistry (IHC; $n=$ 30), wherein patients with greater than median TGFBI immunostaining (cytoplasmic or membrane) had a higher 5 -year OS of 80 vs. $60 \%$ for patients with low TGFBI immunoexpression $(p=0.024$; Fig. 3g, Supplementary Figure S3c), corroborating the clinical significance of TGFBI in mediating NPC survival. 


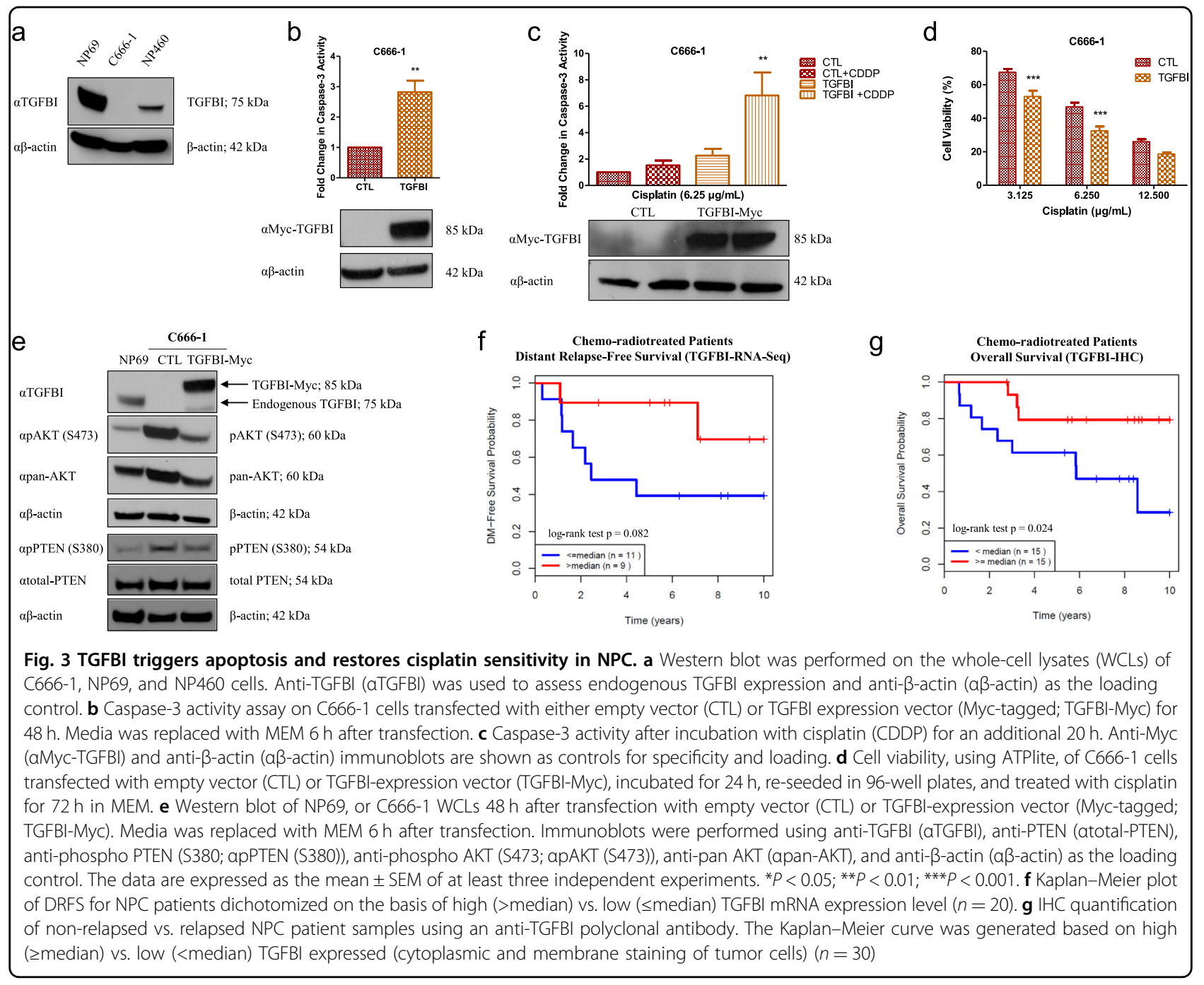

\section{TGFBI downregulation triggers AKT and EMT-like pathways via TGF $\beta$}

A change in cellular morphology was observed during the generation of miR-449b stably transfected cells, wherein NP69-miR-449b cells exhibited a more elongated and mesenchymal-like shape, compared to NP69-miRcontrol cells (Fig. 4a, Supplementary Figure S3d). Thus, the role of EMT in cisplatin resistance was investigated in these models. Because TGF $\beta 1$ is a known inducer of EMT and is associated with TGFBI expression ${ }^{21,32}$, its expression was first evaluated in these cells. Higher intracellular levels of active TGF $\beta 1$ associated with Smad2 phosphorylation were observed in NP69-miR-449b cells, in comparison to the control cells (Fig. 4b, Supplementary Figure S5e). This increase in active intracellular TGF $\beta 1$ was accompanied by increased expression of the transcriptional repressor ZEB1 and decreased expression of the epithelial marker E-cadherin (CDH1) at both the protein (Fig. 4c, Supplementary Figure S5e) and mRNA levels
(Supplementary Figure S3e). Moreover, two other mesenchymal markers vimentin (VIM; mRNA level; Supplementary Figure S3e) and N-cadherin (CDH2; protein and mRNA levels; Fig. 4c, Supplementary Figure S3e, Supplementary Figure S5e) were also upregulated.

Both NP69-shTGFBI cell lines exhibited a similar molecular phenotype: (1) greater levels of active intracellular TGF $\beta 1$ associated with phospho-Smad2 expression (Fig. 4d, Supplementary Figure S5f); (2) higher expression of the mesenchymal markers ZEB1, CDH2 (protein and mRNA levels; Fig. 4e, Supplementary Figure S3f, Supplementary Figure S5f), and VIM (mRNA level; Supplementary Figure S3f); and (3) lower CDH1 expression (protein and mRNA levels; Fig. 4e, Supplementary Figure S3f, Supplementary Figure S5f). Although, these changes were not accompanied by morphological alterations, they suggest that TGFBI downregulation promotes or contributes to an EMT-like phenotype (the transcriptional expression of Snail and Slug, common promoters of 


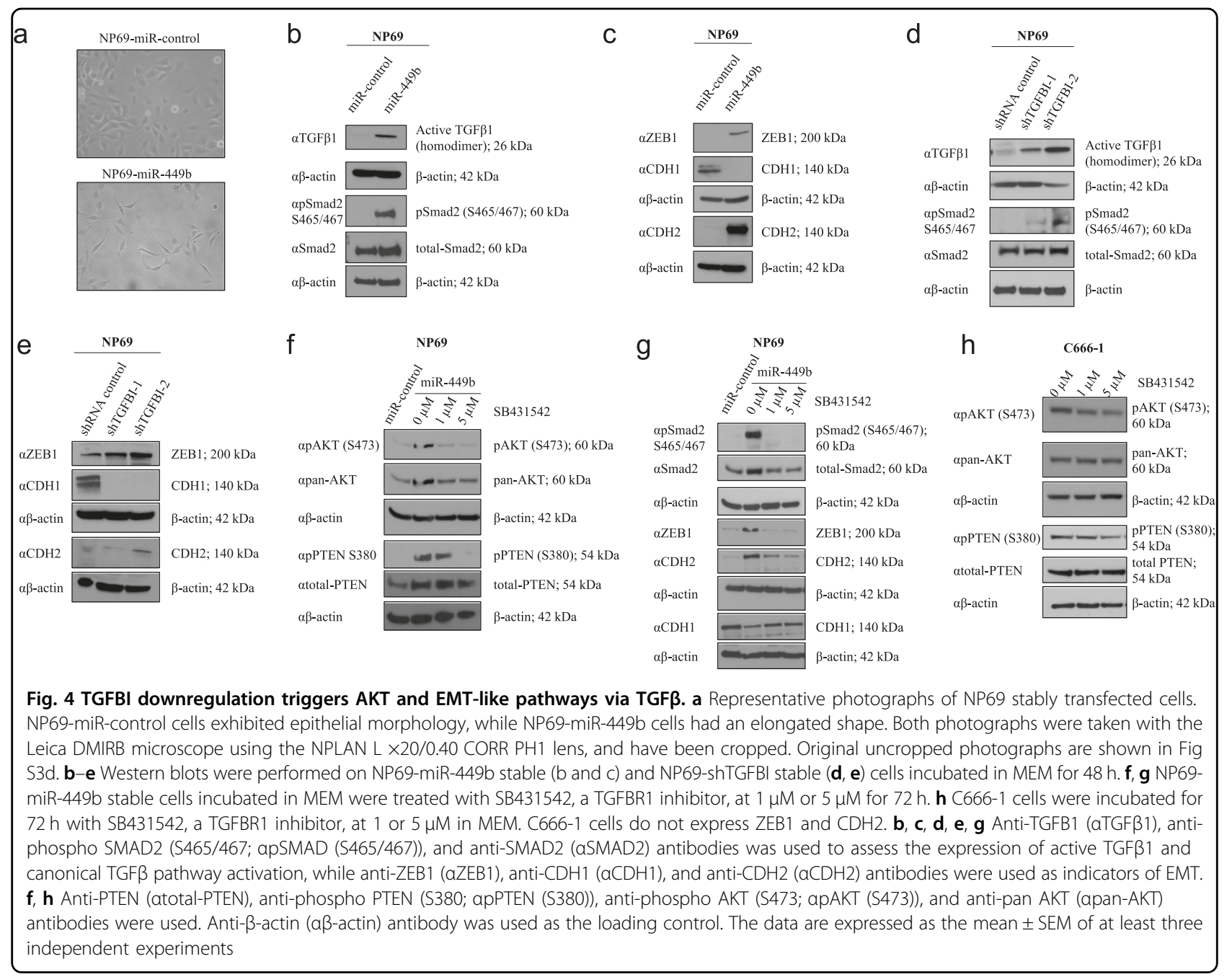

EMT induced by TGF $\beta 1$, were unchanged in both models; data not shown). Transient overexpression of TGFBI in C666-1 cells diminished the intracellular expression of active TGF $\beta 1$ (Supplementary Figure S3g; analysis of EMT-like phenotype was not possible as C666-1 cells do not express ZEB1 and CDH2).

In order to evaluate the significance of active TGF $\beta 1$ expression in our model, NP69-miR-449b cells were treated with the TGFBR1 inhibitor SB431542 (1 and 5 $\mu \mathrm{M})$. This treatment resulted in the reduction of phosphorylated AKT (S473), phosphorylated PTEN (S380) (Fig. 4f, Supplementary Figure S5g), phosphorylated Smad2, ZEB1, and CDH2, whereas CDH1 was slightly upregulated (Fig. 4g, Supplementary Figure S5h), which confirmed previous studies suggesting that the inhibition of PTEN by TGF $\beta 1$ was the cause of TGF $\beta$-dependent AKT activation ${ }^{46,47}$. In addition, C666-1 cells treated with SB431542 reduced AKT phosphorylation (S473) and PTEN phosphorylation (Fig. 4h, Supplementary Figure S5i), a phenotype similar to that observed after TGFBI overexpression (Figs. $2 \mathrm{~g}$ and $3 \mathrm{e}$ ). This further corroborated a significant role of TGF $\beta 1$ in AKT and PTEN phosphorylation, leading to chemoresistance in C666-1 cells. However, treatment of NP69-miR-449b cells with MK-2206 (the AKT inhibitor) did not affect the expression of ZEB1, CDH1, or CDH2 (Supplementary Figure $\mathrm{S} 3 \mathrm{~h})$. Taken together, these data indicate that when TGFBI is downregulated, TGF $\beta 1$ causes: (1) PTEN inhibition and AKT activation; and (2) increased ZEB1 and $\mathrm{CDH} 2$ expression, along with decreased $\mathrm{CDH} 1$. In summary, the absence of TGFBI alone, or its absence mediated by miR-449b, increased chemoresistance partially through the regulation of an EMT-like TGF $\beta 1$-dependent mechanism and through PTEN/AKT regulation in these cellular models of NPC.

\section{TGFBI regulates TGF $\beta$ signaling by binding ITGB3/5}

The binding of pro-TGF $\beta 1$ to integrins has been shown to trigger the release of active TGF $\beta 1^{17,48,49}$, enabling it to bind to its receptors, inducing their phosphorylation, and 


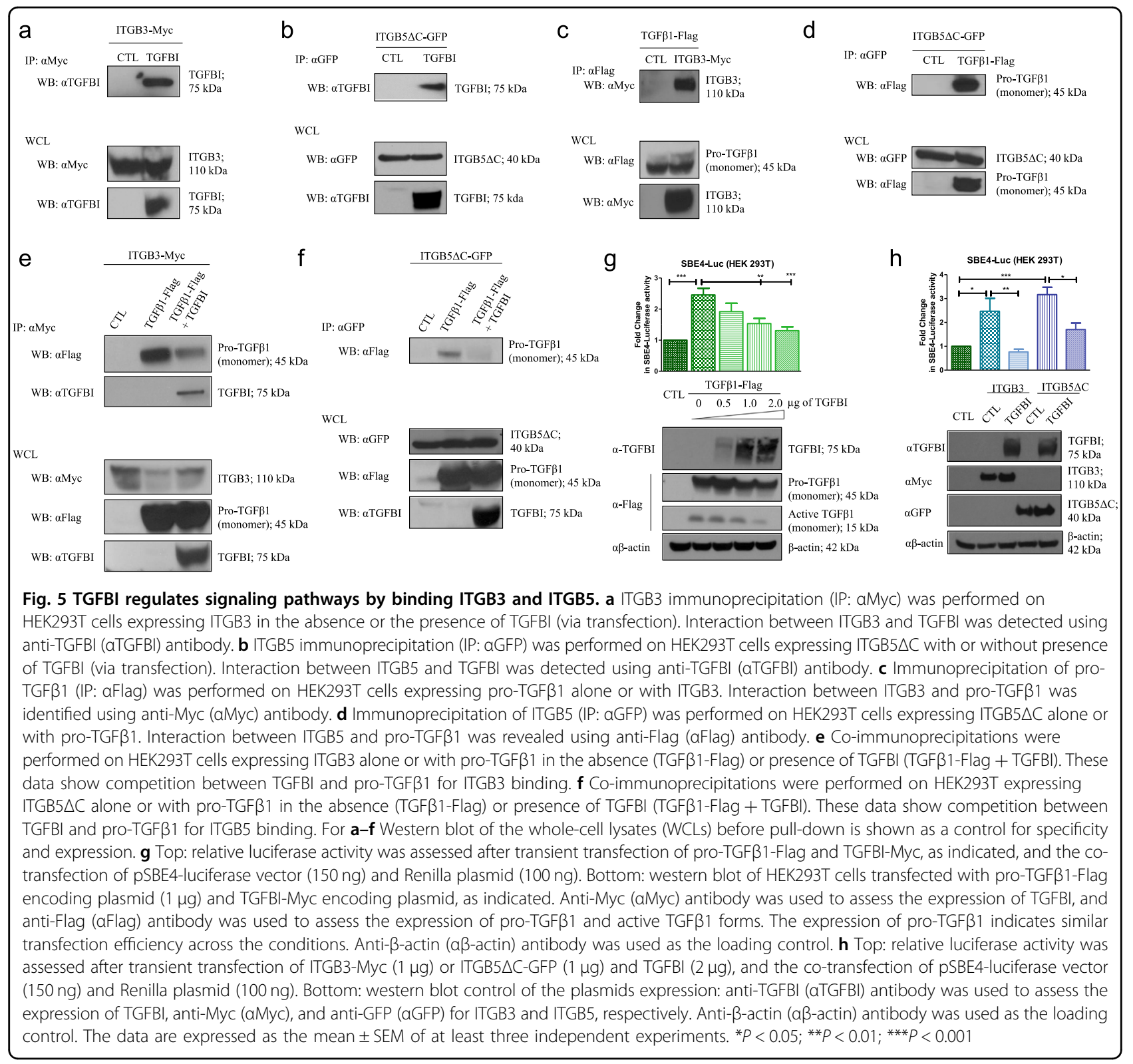

triggering the canonical Smad2/3 and noncanonical signaling pathways ${ }^{18,50}$. Because TGFBI binds to integrin $\alpha v \beta 3 / 5^{19,22-24}$, we hypothesized that TGFBI competes with pro-TGF $\beta 1$ for integrin $\alpha v \beta 3 / 5$ binding, leading to a decrease in TGF $\beta$ signaling when TGFBI is overexpressed, and an increase in TGF $\beta$ signaling when TGFBI is downregulated.

To validate this relationship, the interaction between TGFBI and integrin $\alpha v \beta 3 / 5$ was first confirmed with pulldowns of $\beta 3$ - (ITGB3) and $\beta 5$-integrin (ITGB5) in 293 T cells expressing ITGB3 and ITGB5 $\Delta C$ ( $\beta 5$-integrin without its cytoplasmic tail) (via transfection), respectively (Fig. 5a, b). Secondly, co-immunoprecipitation was used to demonstrate that pro-TGF $\beta 1$ binds to ITGB3 and
ITGB5 $\Delta \mathrm{C}$ (Fig. 5c, d). Finally, overexpression of TGFBI led to a decrease in the interaction between pro-TGF $\beta 1$ and ITGB3/5, confirming that TGFBI does indeed compete with pro-TGF $\beta 1$ for binding to ITGB3/5 (Fig. 5e, f). To our surprise, overexpression of pro-TGF $\beta 1$ did not disrupt the interaction between TGFBI and ITGB3/5 in our model (Supplementary Figure S4a and b), suggesting that TGFBI could have a greater affinity to ITGB3/5 than pro-TGF $\beta 1$.

Ligand-receptor complex internalization has been shown to be an important component of both TGF $\beta$ pathway induction and the molecular turnover of active TGF $\beta 1$ and its receptors ${ }^{51,52}$. Thus, to determine the effects of competition between TGFBI and pro-TGF $\beta 1$, 
we assessed Smad3/4 activity using the pSBE4-luc vector (luciferase vector reporter containing four tandem copies of Smad-binding element) and the transcription of PAI-1 (a well-characterized target of TGF $\beta 1$ ), after cotransfection of pro-TGF $\beta 1$ with increasing amounts of TGFBI. In the absence of TGFBI, the pSBE4-luc vector was activated (Fig. 5g; top) and PAI-1 was transcribed (Supplementary Figure S4c), which corroborated the high levels of active TGF $\beta 1$ observed by western blot (Fig. 5g; bottom). In addition, increasing levels of TGFBI led to a decrease in luciferase activity of the pSBE4-luc vector (Fig. $5 \mathrm{~g}$; top), with a corresponding reduction in the level of intracellular active TGF $\beta 1$ (Fig. $5 \mathrm{~g}$; bottom) and PAI-1 transcription (Supplementary Figure S4c). In our controls, we observed a reduction in luciferase activity of the pSBE4-luc vector after SB431542 treatment (Supplementary Figure S4d; left), with no induction of luciferase activity of the pSBE-luc vector (vector reporter containing one copy of Smad-binding element) after transfection of pro-TGF $\beta 1$ (Supplementary Figure S3d; right). Interestingly, this finding corroborated the observations in Fig. 4b, d, whereby TGFBI repression was accompanied by high intracellular levels of active TGF $\beta 1$ and phosphoSmad2 expression. Additionally, we investigated whether the overexpression of TGFBI could affect the endogenous TGF $\beta 1$ pathway activation induced by exogenous ITGB3/ 5 expression. As seen in Fig. 5h, overexpression of either ITGB3 or ITGB5 $\triangle C$ triggered the induction of the pSBE4-luc vector (but not pSBE-luc (Supplementary Figure S4e; right)), whereas TGFBI expression caused its inhibition. This corroborated our hypothesis of TGF $\beta 1$ pathway inhibition via the binding of TGFBI to ITGB3/5. As a control, SB431542 exposure inhibited the TGF $\beta 1$ pathway activity induced by ITGB3/5 (Supplementary Figure S4e; left). Taken together, these data demonstrate an important role of TGFBI in controlling TGF $\beta 1$ pathway activity via its binding with ITGB3/5.

In summary, TGFBI-ITGB3/5 binding controls AKT phosphorylation via two mechanisms: (1) activation of PTEN via its dephosphorylation; and (2) competition with pro-TGF $\beta 1$ in the ECM. Furthermore, the phenotypic changes observed in this study suggest that the TGFBI-

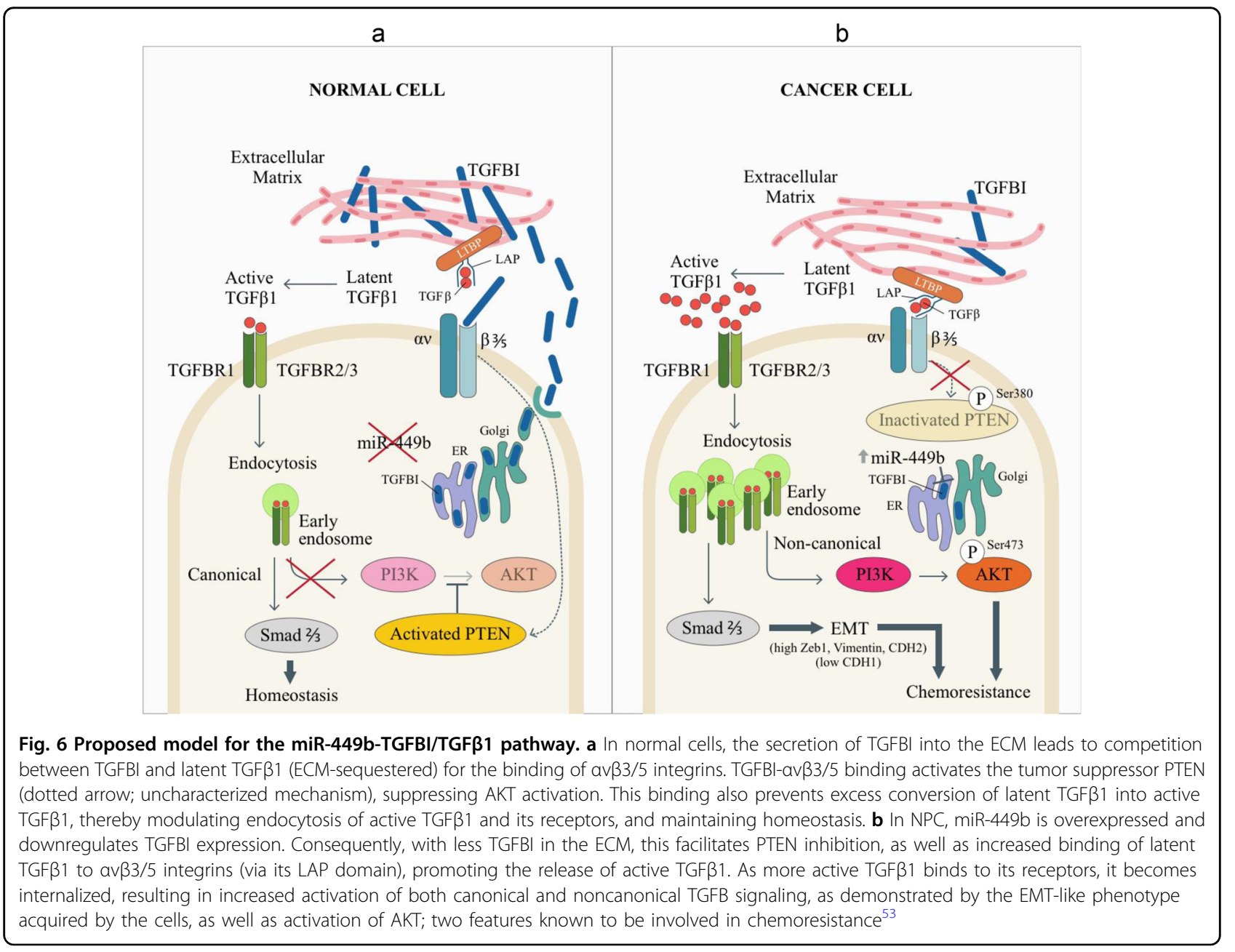


TGF $\beta 1$ balance is critical for TGF $\beta$ pathway activation, which is also concordant with studies reporting the importance of the TGF $\beta$ pathway in EMT and chemoresistance $^{11-14,53}$.

\section{Discussion}

This study has demonstrated the importance of the balance between TGFBI and TGF $\beta 1$ in mediating chemotherapy resistance via the TGF $\beta$ pathway. We showed that overexpression of miR-449b resulted in TGFBI inhibition and reduction of TGFBI secretion, allowing for increased integrin-mediated active TGF $\beta 1$ release, with subsequent TGF $\beta 1$ canonical/noncanonical pathway activation (Fig. 6). Previous studies have reported elevated TGF $\beta 1$ in NPC patient sera ${ }^{54}$, although the source of the secretion remained unclear. Interestingly, two EBV proteins, latent membrane protein 1 and EBV-encoded nuclear antigen (EBNA1), have been reported to abolish canonical TGF $\beta$-Smad signaling ${ }^{55-57}$, while inducing TGF $\beta 1$ expression in vitro ${ }^{56,58}$. A synergistic effect between EBV infection and TGF $\beta 1$ treatment on EMT has also been reported in lung epithelial A549 cells $^{59}$, and several EMT-related genes are elevated in NPC ${ }^{60-62}$.

The interplay between TGFBI and TGF $\beta 1$ that we have identified has additional importance from a regulatory perspective. Treatment with TGF $\beta 1$ may induce the expression of $\mathrm{TGFBI}^{21}$, suggesting a feedback mechanism by which TGF $\beta 1$ might induce apoptosis and prevent further activation. This hypothesis is supported by the fact that pro-TGF $\beta 1$ did not seem to displace TGFBI from binding to ITGB3/5, but this will require further investigation. In addition, EBNA1 has been described as a repressor of canonical TGF $\beta$ signaling, promoting Smad2 turnover and TGFBI downregulation ${ }^{57}$. In our current study, TGFBI expression is suppressed by miR-449b, which in turn, allows the cell to ensure TGF $\beta 1$-mediated survival.

Two pathways responsible for cisplatin resistance resulting from high miR-449b and low TGFBI-expression levels were identified in our cell line models: (1) inactivated PTEN with increased phosphorylated AKT; and (2) increased ZEB1 and N-cadherin with decreased Ecadherin (which commonly induces an EMT-like phenotype $)^{53}$. Although, such phenotypic changes have already been described for both TGFBI and TGF $\beta 1$ independently $^{19,27,45,50}$, the mechanisms linking TGFBI to TGF $\beta 1$ have not been elucidated until now. Here we demonstrated the competition between TGFBI and pro-TGF $\beta 1$ for ITGB3/5 binding, possibly via their RGD domains. Thus, the repression of TGFBI caused by elevated miR$449 \mathrm{~b}$ leads to an imbalance in integrin binding. The paucity of TGFBI allows for increased pro-TGF $\beta 1$ activation via integrin binding. Thus, more active TGF $\beta 1$ will be released, leading to increased activity of the canonical and noncanonical TGF $\beta$ pathways. Further investigation is needed to determine the specific role of ITGB3/5 in TGFBI-dependent apoptosis in NPC. One hypothesis could be that ITGB3/5 serves as an anchor for the cleavage of TGFBI, releasing its $\mathrm{C}$-terminal domain, leading to apoptosis.

This study also identified miR-449b and TGFBI as promising biomarkers for cisplatin response in NPC. The results are consistent with a previous study reporting that TGFBI is a favorable prognostic factor for survival in patients with squamous cell lung cancer treated with adjuvant platinum-based chemotherapy ${ }^{29}$. Future evaluations may also include assaying patient sera levels of TGFBI, given that it is a secreted protein; hence could potentially serve as a circulating biomarker of chemosensitivity. Finally, the importance of the TGFBI-TGF $\beta 1$ balance that we report here for NPC warrants further exploration in other human malignancies.

\section{Materials and methods \\ Cell culture, transfection, and infection}

The human normal nasopharyngeal cell lines, NP69 (immortalized by SV40) and NP460 (immortalized by hTert), were a kind gift from George S.W. Tsao (University of Hong Kong). NP69 cells were maintained in keratinocyte-free media supplemented with EGF and pituitary serum (Invitrogen, Carlsbad, CA, USA); NP460 cells were grown in keratinocyte-free media mixed with Opti-MEM (Invitrogen) at a 1:1 ratio. C666-1 (EBVpositive NPC) cells were cultured in RPMI media supplemented with 10\% FBS. HEK293T, used for functional analysis and virus production, were cultured in DMEM with 10\% FBS. Each new frozen batch was tested for mycoplasma (MycoAlert Mycoplasma Detection Kit, Lonza, Basel, Switzerland); after 10-11 passages, cells were discarded and a new batch was used. NP69 and C666-1 cell lines were authenticated using polymorphic short tandem repeat method and RNA sequencing. Transient transfections for co-immunoprecipitation and virus production were performed using calcium phosphate. JetPRIME (Polyplus-transfection, Graffenstaden, France) was used to transfect C666-1 for cell death assays and functional analysis in HEK293T. pCMV6-TGFBIMyc (Origene, Rockville, MD, USA), pCMV6-XL5-TGFBI non-tagged (Origene), pcDNA3.1-beta-3 (Addgene plasmid \#27289; from Timothy Springer, Addgene, Cambridge, MA, USA), pCX-EGFP beta5 $\Delta C$ (Addgene plasmid \#14996; from Raymond Birge), pCMV3-TGFB1Flag (Sino Biological Inc., Beijing, China), pSBE4-Luc (Addgene plasmid \#16495; from Bert Vogelstein ${ }^{63}$ ), pSBELuc (Addgene plasmid \#16527; from Bert Vogelstein ${ }^{63}$ ), and pcDNA.3.1-Flag were used. NP69 and NP460 cells were transfected with pre-miR-449b (Ambion, Austin, 
TX, USA; $20 \mathrm{nM}$ ), while pre-miR negative control (Ambion; $20 \mathrm{nM}$ ) was transfected using JetPRIME.

Stable cell lines were generated using lentiviral infection. pLV-miRNA-449b (Biosettia, San Diego, CA, USA), pLKO.1 shRNA-TGFBI-1 (TRCN0000062174) and shRNA-TGFBI-2 (TRCN0000062176) (Sigma, St. Louis, MO, USA), and their respective control vectors were used. The plasmids were transfected into HEK293T with psPAX2 and pCMV-VSV-G (Addgene) for virus encapsulation. After an overnight incubation, media was changed, and then at 48 and $72 \mathrm{~h}$ after transfection, the media was collected and changed, centrifuged, and filtered ( $0.45 \mu \mathrm{m}$ filter). NP69 cells were then incubated for $24 \mathrm{~h}$ with each media condition; $48 \mathrm{~h}$ later, cell selection was performed using puromycin or blasticidin.

SB431542 (\#S1067, SelleckChem, Houston, TX, USA) is a selective inhibitor of ALK5, also known as TGF $\beta$ receptor I. MK-2206 (\#S1078, SelleckChem) is a selective inhibitor of AKT1/2/3.

\section{Western blot and co-immunoprecipitation}

For both western blot and co-immunoprecipitation, proteins were extracted using immunoprecipitation buffer (50 mM Hepes pH 7.6, 150 mM NaCl, 5 mM EDTA, 1-2\% Nonidet P-40) in the presence of protease inhibitor cocktail (Roche, Basel, Switzerland), and then separated using a Bolt 4-20\% Gel (Life Technologies, Carlsbad, CA, USA). For immunoprecipitation, the lysates were incubated overnight at $4{ }^{\circ} \mathrm{C}$ either with anti-GFP (Santa Cruz Biotechnology, Dallas, TX, USA), anti-Myc (9E10, Santa Cruz) or anti-Flag M2 (Sigma) antibodies, then incubated for $1 \mathrm{~h}$ with Protein-A Sepharose, and washed three times using immunoprecipitation buffer without NP-40. For western blot, anti-TGFBI (ab155426, Abcam, Cambridge, UK: 1/1 000), anti-pan AKT (40D4, \#2920, Cell Signaling Technology, Danvers, MA, USA: 1/1 000), anti-phospho AKT-S473 (\#9271, Cell Signaling: 1/1 000), anti-PTEN (138G6, \#9559, Cell Signaling: 1/1 000), anti-phospho PTEN S380 (\#9551, Cell Signaling: 1/1 000), anti-TGF $\beta$ (\#3711, Cell Signaling: 1/1 000), anti-Smad2 (D43B4, \#5339, Cell Signaling: 1/1 000), anti-phospho Smad2S465/467 (138D4, \#3108, Cell Signaling: 1/1 000), antip38 MAPK (\#9202, Cell Signaling; 1/1 000), anti-p44/42 MAPK (\#9102, Cell Signaling, 1/1 000), anti-phospho p44/42-Thr202/Tyr204 (\#9101, Cell Signaling, 1/1 000), anti-SAPK/JNK (56G8, \#9258, Cell Signaling, 1/1 000), anti-phospho SAPK/JNK-Thr183/Tyr185 (\#9251, Cell Signaling, 1/1 000), anti-Myc (Santa Cruz: $1 / 5$ 000), antiFlag M2 (Sigma: $1 / 5$ 000), and anti- $\beta$-actin (Sigma: $1 / 5$ 000) antibodies were used. Please note that the phosphorylated form of the MAPK proteins required the use of the SuperSignal West Femto ECL (Pierce, \#34095), whereas all other proteins were detected using normal Pierce ECL (\#32209).
IHC

Formalin-fixed and paraffin-embedded (FFPE) tumor sections were stained after microwave antigen retrieval using $0.01 \mathrm{M}$ citric acid ( $\mathrm{pH}$ 6.0) in combination with LSAB + System-HRP (Dako, Les Ulis, France). Rabbit polyclonal anti-TGFBI (HPA-017019, Sigma: 1/300) antibody was used. Primary antibody was omitted as a negative control. Tumor cytoplasmic staining was scored according to intensity of immunoexpression, with $0,1,2$, and 3 indicating negative, weak, moderate, and strong staining of tumor cells, respectively. IHC scoring was performed blinded, without prior knowledge of clinicopathological parameters; each tissue section was scored twice and the result was then averaged.

\section{qRT-PCR}

RNA was isolated using the Total RNA Purification Kit (Norgen Biotek, Thorold, Canada). One microgram of the total RNA was reverse-transcribed using the iScript cDNA Synthesis Kit (BioRad, Hercules, CA, USA). qRTPCR was performed using either the SYBR Green PCR Master Mix (Life Technologies) or the SYBR Green (Roche) with the following primers: TGFBI (Forward: GTCCACAGCCATTGACCTTT, Reverse: GAGTTTCCAGGGTCTGTCCA); PTEN (Forward: TTGGCGGTGTCATAATGTCT, Reverse: GCAGAAAGACTTGAAGGCGTA); PAI-1 (Forward: GGCCATTACTACGACATCCTG, Reverse: GGTCATGTTGCCTTTCCAGT); ZEB1 (Forward: GTTCTGCCAACAGTTGGTTT, Reverse: GCTCAAGACTGTAGTTGATG); VIM (Forward: TGACCTCTCTGAGGCTGCCAACC, Reverse: TTCCATCTCACGCATCTGGCGCTC); CDH1 (Forward: CACCCTGGCTTTGACGCCGA, Reverse: AAAATTCACTCTGCCCAGGACGCG); CDH2 (Forward: CGCCATCCAGACCGACCCAA, Reverse: GTCGATTGGTTTGACCACGGTGAC); $\beta$-actin (Forward: AGAGCTACGAGCTGCCTGAC, Reverse: AGCACTGTGTTGGCGTACAG); GAPDH (Forward: TGTTGCCATCAATGACCCCTT, Reverse: CTCCACGACGTACTCAGCG); and HPRT (Forward: ATGAACCAGGTTATGACCTTGAT, Reverse: CCTGTTGACTGGTCATTACAATA). The $2^{-\Delta \Delta} \mathrm{Ct}$ method was used to calculate the relative miRNA expression $^{64}$. The mRNA expression levels were normalized to the average expression of three housekeeping genes ( $\beta$-actin, GAPDH, and HPRT).

\section{Proliferation, viability, caspase-3 activity, and clonogenic assays}

For cell viability, the cells were plated at a density of 2000 cells per well in 96-well plates. Cell viability was evaluated using the ATPlite 1 Step Luminescence Assay System (PerkinElmer, Waltham, MA, USA). To assess 
cisplatin resistance, cells were exposed to varying concentrations of cisplatin (CDDP) for $72 \mathrm{~h}$. Caspase-3 activity was assayed $20 \mathrm{~h}$ after cisplatin treatment and/or $48 \mathrm{~h}$ after transfection using the Caspase-3 Fluorometric Kit (BioVision, Milpitas, CA, USA).

For clonogenic assays, 500 cells per well were seeded in 6-well plates and incubated for $24 \mathrm{~h}$, then treated with cisplatin at varying doses. After 10 days, colonies $(>50$ cells) were fixed with methanol and stained with crystal violet, then counted. It is important to note that C666-1 cells are not suitable for clonogenic assays as they do not form colonies; only NP69 cells were used for this assay.

\section{Luciferase reporter assay}

MiR-449b/TGFBI binding activity: the $3^{\prime}$ untranslated region ( $3^{\prime} \mathrm{UTR}$ ) of TGFBI containing the putative wildtype (WT) target site for miR-449b was cloned into the pMIR-REPORT vector (Ambion) (pMIR-TGFBI 3'UTR $\mathrm{WT}=$ CTAGTGCTCAGCGGATGTCACTGCCTGACATTCA). A mutant sequence was also generated (pMIR-TGFBI 3'UTR Mut=CTAGTGCTCAGCGGATGTGTGACGGAGACATTCA). HEK293T cells were reverse transfected with pre-miR-control and premiR-449b using JetPRIME; $24 \mathrm{~h}$ later, pMIR-TGFBI $3^{\prime}$ UTR WT and pMIR-TGFBI 3'UTR Mut plasmids were co-transfected with pRL-SV Renilla vector (Promega, Madison, WI, USA), also using JetPRIME. After another $24 \mathrm{~h}$, firefly and Renilla luciferase activities were measured using the Dual-Luciferase Reporter Assay (Promega).

TGF $\beta$ activity pathway: HEK293T was co-transfected with pSBE4-Luc vector or pSBE-Luc vector and pRL-SV Renilla vector, in addition to either pro-TGF $\beta 1$, TGFBI, ITGB3, ITGB5 $\Delta$ C, or control plasmids, as indicated in the figure legends.

\section{Patient samples}

Approval for the study was obtained from the Institutional Research Ethics Board (REB) at the Princess Margaret (PM) Cancer Center. Diagnostic FFPE blocks were collected for NPC patients treated at the PM between 1993 and 2009 and the RNA was extracted, as previously described $^{36}$.

\section{RNA-seq and data analysis}

For each NPC sample, $200 \mathrm{ng}$ of input RNA was prepared using the Ribo-Zero Gold rRNA Removal Kit (Illumina, San Diego, CA, USA), followed by library preparation using the TruSeq Stranded Total RNA Sample Prep Kit (Illumina). Libraries were sequenced to $>100$ million paired-end $100 \mathrm{bp}$ reads on the Illumina HiSeq 2000. The reads were then aligned using $\operatorname{STAR}^{65}$ (v2.4.2a), and the expression values were summarized using $\operatorname{RSEM}^{66}$ (v1.2.21).

\section{Statistical analyses}

The statistical significance between groups was determined using either the ANOVA test, followed by the Bonferroni post-test when applicable or the Mann-Whitney $U$-test (socscistatistics.com). Data were represented as the mean \pm SEM. All experiments were performed at least three times using new frozen batches of cells to maintain independence between replicates. Analysis and graphs were completed using GraphPad Prism software.

\section{Acknowledgements}

This research was funded by the Canadian Institutes for Health Research, the Mariano Elia Chair in Head \& Neck Cancer Research, the Campbell Family Institute for Cancer Research, the Ministry of Health and Long-Term Care, and the Princess Margaret Cancer Centre Head \& Neck Translational Program, with philanthropic funds from the Wharton Family, Joe's Team, and Gordon Tozer.

\section{Author details}

${ }^{1}$ Princess Margaret Cancer Centre, University Health Network, Toronto, ON, Canada. ${ }^{2}$ Department of Medical Biophysics, University of Toronto, Toronto, ON, Canada. ${ }^{3}$ LabEx DEVweCAN, Université de Lyon, F-69000 Lyon, France. ${ }^{4}$ Department of Radiation Oncology, Princess Margaret Cancer Centre and University of Toronto, Toronto, ON, Canada. ${ }^{5}$ Division of Radiation Oncology, National Cancer Centre, Singapore, Singapore. ${ }^{6}$ Duke-NUS Graduate School, Singapore, Singapore

\section{Authors' contributions}

P.-A.B., K.W.Y., and F.-F.L. designed the experiments; P.-A.B., J.H.L., W.S., and A.R. performed the experiments; J.P.B. performed bioinformatic analysis; M.L.K.C. supplied clinical samples and data; P.-A.B., J.H.L., J.P.B., W.S., K.W.Y., and F.-F.L. analyzed the data; P.-A.B., J.H.L., K.W.Y., and F.-F.L. wrote the manuscript. All authors read and approved the manuscript.

\section{Conflict of interest}

The authors declare that they have no conflict of interest.

\section{Publisher's note}

Springer Nature remains neutral with regard to jurisdictional claims in published maps and institutional affiliations.

Supplementary Information accompanies this paper at https://doi.org/ 10.1038/s41389-018-0050-x.

Received: 7 September 2017 Revised: 26 February 2018 Accepted: 11 April 2018

Published online: 22 May 2018

\footnotetext{
References

1. Raab-Traub, N. Epstein-Barr virus and nasophanyngeal carcinoma. Semin. Cancer Biol. 3, 297-307 (1992).

2. Raab-Traub, N. Epstein-Barr virus in the pathogenesis of NPC. Semin. Cancer Biol. 12, 431-441 (2002).

3. Lo, K. W. To, K. F. \& Huang, D. P. Focus on nasophanyngeal carcinoma. Cancer Cell 5, 423-428 (2004).

4. Ferlay, J. et al. Cancer incidence and mortality worldwide: sources, methods and major patterns in GLOBOCAN 2012. Int. J. Cancer 136, E359-E386 (2015).

5. Lee, A. W. et al. Preliminary results of a randomized study (NPC-9902 Trial) on therapeutic gain by concurrent chemotherapy and/or accelerated fractionation for locally advanced nasopharyngeal carcinoma. Int J. Radiat. Oncol. Biol. Phys. 66, 142-151 (2006).

6. Lee, A. W. et al. Factors contributing to the efficacy of concurrent-adjuvant chemotherapy for locoregionally advanced nasopharyngeal carcinoma:
} 
combined analyses of NPC-9901 and NPC-9902 Trials. Eur. J. Cancer 47, 656-666 (2011).

7. Huncharek, M. \& Kupelnick, B. Combined chemoradiation versus radiation therapy alone in locally advanced nasopharyngeal carcinoma: results of a meta-analysis of 1,528 patients from six randomized trials. Am. J. Clin. Oncol. 25, 219-223 (2002)

8. Lo, K. W. \& Huang, D. P. Genetic and epigenetic changes in nasophanyngeal carcinoma. Semin. Cancer Biol. 12, 451-462 (2002).

9. Lee, A. W. et al. The battle against nasopharyngeal cancer. Radiother. Oncol. 104, 272-278 (2012).

10. Lee, A. W. et al. Evolution of treatment for nasopharyngeal cancer--success and setback in the intensity-modulated radiotherapy era. Radiother. Oncol. 110, 377-384 (2014).

11. Zheng, $X$. et al. Epithelial-to-mesenchymal transition is dispensable for metastasis but induces chemoresistance in pancreatic cancer. Nature 527, 525-530 (2015).

12. Fischer, K. R. et al. Epithelial-to-mesenchymal transition is not required for lung metastasis but contributes to chemoresistance. Nature 527, 472-476 (2015).

13. Akhurst, R. J. \& Hata, A. Targeting the TGF $\beta$ signalling pathway in disease. Nat. Rev. Drug Discov. 11, 790-811 (2012).

14. Oshimori, N., Oristian, D. \& Fuchs, E. TGF- $\beta$ promotes heterogeneity and drug resistance in squamous cell carcinoma. Cell 160, 963-976 (2015).

15. ten Dijke, P. \& Arthur, H. M. Extracellular control of TGFbeta signalling in vascular development and disease. Nat. Rev. Mol. Cell Biol. 8, 857-869 (2007)

16. Ludbrook, S. B., Barry, S. T., Delves, C. J. \& Horgan, C. M. The integrin alphavbeta3 is a receptor for the latency-associated peptides of transforming growth factors beta1 and beta3. Biochem. J. 369, 311-318 (2003).

17. Wipff, P. J. \& Hinz, B. Integrins and the activation of latent transforming growth factor beta1 - an intimate relationship. Eur. J. Cell Biol. 87, 601-615 (2008).

18. Massagué, J. TGF $\beta$ signalling in context. Nat. Rev. Mol. Cell Biol. 13, 616-630 (2012).

19. Son, H. N., Nam, J. O., Kim, S. \& Kim, I. S. Multiple FAS1 domains and the RGD motif of TGFBI act cooperatively to bind alphavbeta3 integrin, leading to antiangiogenic and anti-tumor effects. Biochim. Biophys. Acta 1833, 2378-2388 (2013).

20. Lee, B. H. et al. betaig-h3 triggers signaling pathways mediating adhesion and migration of vascular smooth muscle cells through alphavbeta5 integrin. Exp. Mol. Med. 38, 153-161 (2006).

21. Skonier, J. et al. cDNA cloning and sequence analysis of beta ig-h3, a novel gene induced in a human adenocarcinoma cell line after treatment with transforming growth factor-beta. DNA Cell Biol. 11, 511-522 (1992).

22. Kim, J. E. et al. Identification of motifs in the fasciclin domains of the transforming growth factor-beta-induced matrix protein betaig-h3 that interact with the alphavbeta5 integrin. J. Biol. Chem. 277, 46159-46165 (2002).

23. Nam, J. O. et al. Identification of the alphavbeta3 integrin-interacting motif of betaig-h3 and its anti-angiogenic effect. J. Biol. Chem. 278, 25902-25909 (2003).

24. Nam, J. O., Jeong, H. W., Lee, B. H., Park, R. W. \& Kim, I. S. Regulation of tumor angiogenesis by fastatin, the fourth FAS1 domain of betaig-h3, via alphavbeta3 integrin. Cancer Res. 65, 4153-4161 (2005).

25. Ween, M. P., Oehler, M. K. \& Ricciardelli, C. Transforming growth factor-betainduced protein (TGFBI)/( (Big-H3): a matrix protein with dual functions in ovarian cancer. Int. J. Mol. Sci. 13, 10461-10477 (2012).

26. Zhang, Y. et al. TGFBI deficiency predisposes mice to spontaneous tumor development. Cancer Res. 69, 37-44 (2009).

27. Ahmed, A. A. et al. The extracellular matrix protein TGFBI induces microtubule stabilization and sensitizes ovarian cancers to paclitaxel. Cancer Cell $\mathbf{1 2}$ 514-527 (2007).

28. Irigoyen, M. et al. TGFBI expression is associated with a better response to chemotherapy in NSCLC. Mol. Cancer 9, 130 (2010).

29. Pajares, M. J. et al. TGFBl expression is an independent predictor of survival in adjuvant-treated lung squamous cell carcinoma patients. Br. J. Cancer $\mathbf{1 1 0}$ 1545-1551 (2014).

30. Wang, N. et al. TGFBI promoter hypermethylation correlating with paclitaxel chemoresistance in ovarian cancer. J. Exp. Clin. Cancer Res. 31, 6 (2012).

31. Wen, G. et al. TGFBI expression reduces in vitro and in vivo metastatic potential of lung and breast tumor cells. Cancer Lett. 308, 23-32 (2011).

32. Skonier, J. et al. beta ig-h3: a transforming growth factor-beta-responsive gene encoding a secreted protein that inhibits cell attachment in vitro and suppresses the growth of $\mathrm{CHO}$ cells in nude mice. DNA Cell Biol. 13, 571-584 (1994).
33. Kim, J. E. et al. RGD peptides released from beta ig-h3, a TGF-beta-induced cell-adhesive molecule, mediate apoptosis. Oncogene 22, 2045-2053 (2003).

34. Zhu, J., Chen, X., Liao, Z., He, C. \& Hu, X. TGFBI protein high expression predicts poor prognosis in colorectal cancer patients. Int J. Clin. Exp. Pathol. 8, 702-710 (2015).

35. Lebdai, S. et al. Identification and validation of TGFBI as a promising prognosis marker of clear cell renal cell carcinoma. Urol. Oncol. 33, 69 e11-68 (2015).

36. Bruce, J. P. et al. Identification of a microRNA signature associated with risk of distant metastasis in nasopharyngeal carcinoma. Oncotarget 6, 4537-4550 (2015).

37. Zhang, P. et al. MicroRNA-10b regulates epithelial-mesenchymal transition by modulating KLF4/Notch1/E-cadherin in cisplatin-resistant nasopharyngeal carcinoma cells. Am. J. Cancer Res. 6, 141-156 (2016).

38. Zhang, H., Cao, H., Xu, D. \& Zhu, K. MicroRNA-92a promotes metastasis of nasopharyngeal carcinoma by targeting the PTEN/AKT pathway. Onco Targets Ther. 9, 3579-3588 (2016).

39. Zhen, Y. et al. miR-374a-CCND1-pPI3K/AKT-c-JUN feedback loop modulated by PDCD4 suppresses cell growth, metastasis, and sensitizes nasopharyngeal carcinoma to cisplatin. Oncogene 36, 275-285 (2016).

40. Alajez, N. M. et al. MiR-218 suppresses nasopharyngeal cancer progression through downregulation of survivin and the SLIT2-ROBO1 pathway. Cancer Res. 71, 2381-2391 (2011).

41. Blanchard, P., Ribassin-Majed, L., Lee, A. \& Pignon, J. P. Comment on "Chemoradiotherapy regimens for locoregionally advanced nasophanyngeal carcinoma: a Bayesian network meta-analysis", published in Eur J Cancer 51 (2015), 1570-1579. Eur. J. Cancer 56, 183-185 (2016).

42. Chou, J. et al. Nasopharyngeal carcinoma--review of the molecular mechanisms of tumorigenesis. Head Neck 30, 946-963 (2008).

43. Vazquez, F., Ramaswamy, S., Nakamura, N. \& Sellers, W. R. Phosphorylation of the PTEN tail regulates protein stability and function. Mol. Cell Biol. 20, 5010-5018 (2000).

44. Ross, A. H. \& Gericke, A. Phosphorylation keeps PTEN phosphatase closed for business. Proc. Natl Acad. Sci. USA 106, 1297-1298 (2009).

45. Wen, G. et al. Transforming growth factor- $\beta$-induced protein (TGFBI) suppresses mesothelioma progression through the Akt/mTOR pathway. Int. J. Oncol. 39, 1001-1009 (2011).

46. Aoyama, D. et al. Involvement of TGF $\beta$-induced phosphorylation of the PTEN C-terminus on TGF $\beta$-induced acquisition of malignant phenotypes in lung cancer cells. PLoS ONE 8, e81133 (2013).

47. Chow, J. Y., Cabral, J. A.r Chang, J. \& Carethers, J. M. TGFbeta modulates PTEN expression independently of SMAD signaling for growth proliferation in colon cancer cells. Cancer Biol. Ther. 7, 1694-1699 (2008).

48. Hinz, B. It has to be the av: myofibroblast integrins activate latent TGF- $\beta 1$. Nat. Med. 19, 1567-1568 (2013).

49. Dong, X. et al. Force interacts with macromolecular structure in activation of TGF-B. Nature 542, 55-59 (2017).

50. Massagué, J. \& Xi, Q. TGF- $\beta$ control of stem cell differentiation genes. FEBS Lett. 586, 1953-1958 (2012).

51. Di Guglielmo, G. M., Le Roy, C., Goodfellow, A. F. \& Wrana, J. L. Distinct endocytic pathways regulate TGF-beta receptor signalling and turnover. Nat. Cell Biol. 5, 410-421 (2003).

52. Hayes, S., Chawla, A. \& Corvera, S. TGF beta receptor internalization into EEA1enriched early endosomes: role in signaling to Smad2. J. Cell Biol. 158 1239-1249 (2002).

53. Singh, A. \& Settleman, J. EMT, cancer stem cells and drug resistance: an emerging axis of evil in the war on cancer. Oncogene 29, 4741-4751 (2010).

54. Xu, J., Menezes, J., Prasad, U., SpringerAmpamp; Ahmad, A. Elevated serum levels of transforming growth factor beta1 in Epstein-Barr virus-associated nasophanyngeal carcinoma patients. Int. J. Cancer 84, 396-399 (1999).

55. Lo, A. K., Dawson, C. W., Lo, K. W., Yu, Y. \& Young, L. S. Upregulation of Id1 by Epstein-Barr virus-encoded LMP1 confers resistance to TGFbeta-mediated growth inhibition. Mol. Cancer 9, 155 (2010).

56. Morris, M. A. et al. The Epstein-Barr virus encoded LMP1 oncoprotein modulates cell adhesion via regulation of activin AVTGF $\beta$ and $\beta 1$ integrin signalling. Sci. Rep. 6, 19533 (2016).

57. Wood, V. H. et al. Epstein-Barr virus-encoded EBNA1 regulates cellular gene transcription and modulates the STAT1 and TGFbeta signaling pathways. Oncogene 26, 4135-4147 (2007).

58. Wang, L. et al. Epstein-Barr virus nuclear antigen 1 (EBNA1) protein induction of epithelial-mesenchymal transition in nasopharyngeal carcinoma cells. Cancer 120, 363-372 (2014). 
59. Sides, M. D. et al. The Epstein-Barr virus latent membrane protein 1 and transforming growth factor-- $\beta 1$ synergistically induce epithelial--mesenchymal transition in lung epithelial cells. Am. J. Respir. Cell Mol. Biol. 44, 852-862 (2011).

60. Cao, S. et al. Upregulation of flotillin-1 promotes invasion and metastasis by activating TGF- $\beta$ signaling in nasopharyngeal carcinoma. Oncotarget 7, 4252-4264 (2016).

61. Zhao, L. et al. Flotillin-2 promotes nasopharyngeal carcinoma metastasis and is necessary for the epithelial-mesenchymal transition induced by transforming growth factor- $\beta$. Oncotarget 6, 9781-9793 (2015).

62. Xia, Y. Y. et al. HMGA2 is associated with epithelial-mesenchymal transition and can predict poor prognosis in nasopharyngeal carcinoma. Onco Targets Ther. 8, 169-176 (2015).
63. Zawel, L. et al. Human Smad3 and Smad4 are sequence-specific transcription activators. Mol. Cell 1, 611-617 (1998).

64. Livak, K. J. \& Schmittgen, T. D. Analysis of relative gene expression data using real-time quantitative PCR and the 2(-Delta Delta C(T)) Method. Methods 25 , 402-408 (2001).

65. Dobin et al. STAR: ultrafast universal RNA-seq aligner. Bioinformatics 29, 15-21 (2013).

66. Li, B. \& Dewey, C. N. RSEM: accurate transcript quantification from RNA-Seq data with or without a reference genome. BMC Bioinform. 12, 323 (2011). 